\title{
A proposed role for non-junctional transverse tubules in skeletal muscle as flexible segments allowing expansion of the transverse network
}

\author{
Manuela Lavorato (1), Ramesh Iyer (2), Clara Franzini-Armstrong (1) \\ (1) Department of Cell and Developmental Biology, University of Pennsylvania, Philadelphia, \\ PA, USA; (2) Division of Cardiology, Children Hospital of Philadelphia, Philadelphia, PA, USA
}

This article is distributed under the terms of the Creative Commons Attribution Noncommercial License (CC BY-NC 4.0) which permits any noncommercial use, distribution, and reproduction in any medium, provided the original author(s) and source are credited.

\begin{abstract}
Using a variety of technical approaches, we have detected the presence of continuous triads that cover the entire length of $\mathrm{T}$ tubules in the main white body muscles of several small fish. This is in contrast to the discontinuous association of sarcoplasmic reticulum with $T$ tubules in the red muscles from the same fish as well as in all other previously described muscles in a large variety of skeletal muscles. We suggest that continuous triads are permissible only in muscle fibers that are not normally subject to significant changes in sarcomere length during normal in vivo activity, as is the case for white muscles in the trunk of fish.
\end{abstract}

Key Words: Ultrastructure, sarcoplasmic reticulum, transverse tubules, triads.

Eur J Transl Myol 29 (2): 86-91, 2019

Skeletal muscle fibers are crossed at periodic intervals (either at the level of $\mathrm{Z}$ lines or at the A-I junctions), by networks of transverse $(\mathrm{T})$ tubules that are associated at frequent intervals with elements of the sarcoplasmic reticulum (SR). Andersson-Cedergren ${ }^{1,2}$ was the first to recognize the continuity of the transversely oriented $\mathrm{T}$ tubule network and named it. Functional connections between $\mathrm{T}$ and $\mathrm{SR}$ are at triads (or occasionally dyads), where two (or one) SR elements flank the central T tubules, constituting the sites of excitation-contraction (e-c) coupling. Usually the SR forms extensively interwoven sleeves around the myofibrils, but its functional contacts with the $\mathrm{T}$ tubules are intermittent. As a result, the $\mathrm{T}$ tubule networks are divided into contiguous "junctional" segments (those that are sandwiched between SR elements in triads/dyads) and "free" segments between triads. The "Golgi stain" technique used by Veratti in Pavia at the beginning of the twentieth century and later introduced into electron microscopy, ${ }^{3}$ provides detailed views of $\mathrm{T}$ tubule shapes. ${ }^{4,5,6}$ The junctional tubule segments (jT) have the shape of flat ribbons and follow a straight path between two extended junctional cisternae of the SR, while the free segments (fT) have a smaller, circular cross section and tend to be wavy, so that they are longer than the distance between adjacent triads. This extra length is critical for our subsequent hypothesis. Comparative morphometry studies have established a clear relationship between the frequency and size of the T-SR contacts and the fiber type properties, particularly the rapidity of activation. ${ }^{5,6}$ However, even in the fastest type of muscle fibers such as those responsible for the vibration of toadfish swimbladder during mating, ${ }^{7}$ the measured junctional portion of $\mathrm{T}$ tubule does not exceed $80-90 \%$ of the tubule length. This leaves free $\mathrm{T}$ tubule segments intercalated between the triads. Several superfast muscles, have been imaged by electron microscopy: swimbladder muscles in toadfish, ${ }^{7,8}$ and midshipman; $;^{9,10,11}$ the rattle snake muscle; ${ }^{12,13}$ the ultrasound producing muscle of bats, ${ }^{14}$ and in all cases they show some discontinuity in the $\mathrm{T}$ tubule coverage by SR. All these muscles are responsible for repetitive contractions, of little force and shortening, that induce sound producing vibrations.

\section{Materials and Methods}

We used two experimental approaches. For freezefracture deep-etch, small glassfish (Parambassis ranga, previously known as Chanda ranga) were decapitated, skinned and immersed in 3\% glutaraldehyde in $0.1 \mathrm{M}$ cacodylate buffer, $\mathrm{pH} \sim 7.2$ at room temperature. Small tissue blocks were infiltrated with $40 \%$ methanol with $3 \%$ glycerol, and frozen in Freon. They were freezefractured at $-110^{\circ} \mathrm{C}$ and etched for $15 \mathrm{~min}$ at $-100^{\circ} \mathrm{C}$ and $2.10^{-6}$ Torr in a Balzer's 410 machine. After deepetching, the specimens were cooled to $-150^{\circ} \mathrm{C}$, and rotary shadowed with platinum at an angle of $25^{\circ}$ and replicated 


\section{Role of non-junctional transverse tubules}

Eur J Transl Myol 29 (2): 86-91, 2019

with carbon. The replicas were cleaned in chlorox and examined in a Philips 410 electron microscope (Philips Electron Optics, Mahwah, NJ). ${ }^{15-19}$ In all cases we examined the bulk of the body musculature, ignoring fins and the caudal region of the tail. For SEM images we used the fracturing-maceration technique developed by Tanaka and Mitsushima, ${ }^{20}$ who used it to produce stunning images of rat myocardium. ${ }^{21}$ Adult zebrafish (Danio rerio) and a and a small feeder fish of undefined classification were decapitated, skinned and fixed without further dissection by immersion in $1 \%$ glutaraldehyde and $0.5 \%$ paraformaldehyde in $0.1 \mathrm{M}$ cacodylate buffer, $\mathrm{pH} \sim 7.2$ at room temperature for $1 \mathrm{hr}$, then maintained at $4^{\circ} \mathrm{C}$ for longer periods. The fixed samples were infiltrated with $30 \%$ glycerol, frozen in liquid nitrogen-cooled propane, placed over a cooled plastic surface and immediately fractured by a sharp tap on a previously cooled razor blade that was positioned with the cutting edge parallel to the lateral line and held vertical. The resulting fracture planes were quite variable because the blade tended to slip (see supplemental information). The pieces were thawed and rinsed in $0.1 \mathrm{M}$ cacodylate buffer then postfixed for 1 hour in 1-2\% OsO4 and macerated for $96 \mathrm{hrs}$ in $2-3$ changes of $0.1 \% \mathrm{OsO} 4$ in the same buffer. In order to infiltrate the tissue with $\mathrm{OsO} 4$ to increase its conductivity and thus avoid electrostatic charging of the block during observation in the SEM, the samples were alternatively exposed for $15 \mathrm{~min}$ each to three changes of buffered $2 \%$ OsO4 and $0.5 \%$ low molecular weight tannic acid with extensive washes in between. Finally the tissue fragments were dehydrated in $\mathrm{EtOH}$, critical point dried, sputter coated with gold palladium and examined in a Quanta SEM.

\section{Supplemental information}

The small fish were not pre-dissected. Identification of the muscles' origin was obtained from indirect clues in the fractured specimens. In the case of the glass fish used for deep-etch images fracture was performed in large strips of muscle cut from the central region of the body and the fracture was guided by the microtome arrangement of the freeze-fracture machine So the factures clearly involved only the white body muscles. In the case of specimens prepared for SEM, the fracture was performed on intact segments of the fish. The fracturing razor blade was positioned close to the lateral line, but it easily slipped during the fracturing. Some fracture surfaces broke through the center myotomes which are composed of white fibers. The fractured bundles were also recognized as made up of white fibers by their relatively large fractured surfaces and the scarcity of mitochondria. ${ }^{21}$ Irregularly fractured small pieces were derived from fractures at the edge of the fish, They showed groups of fibers rich in mitochondria that

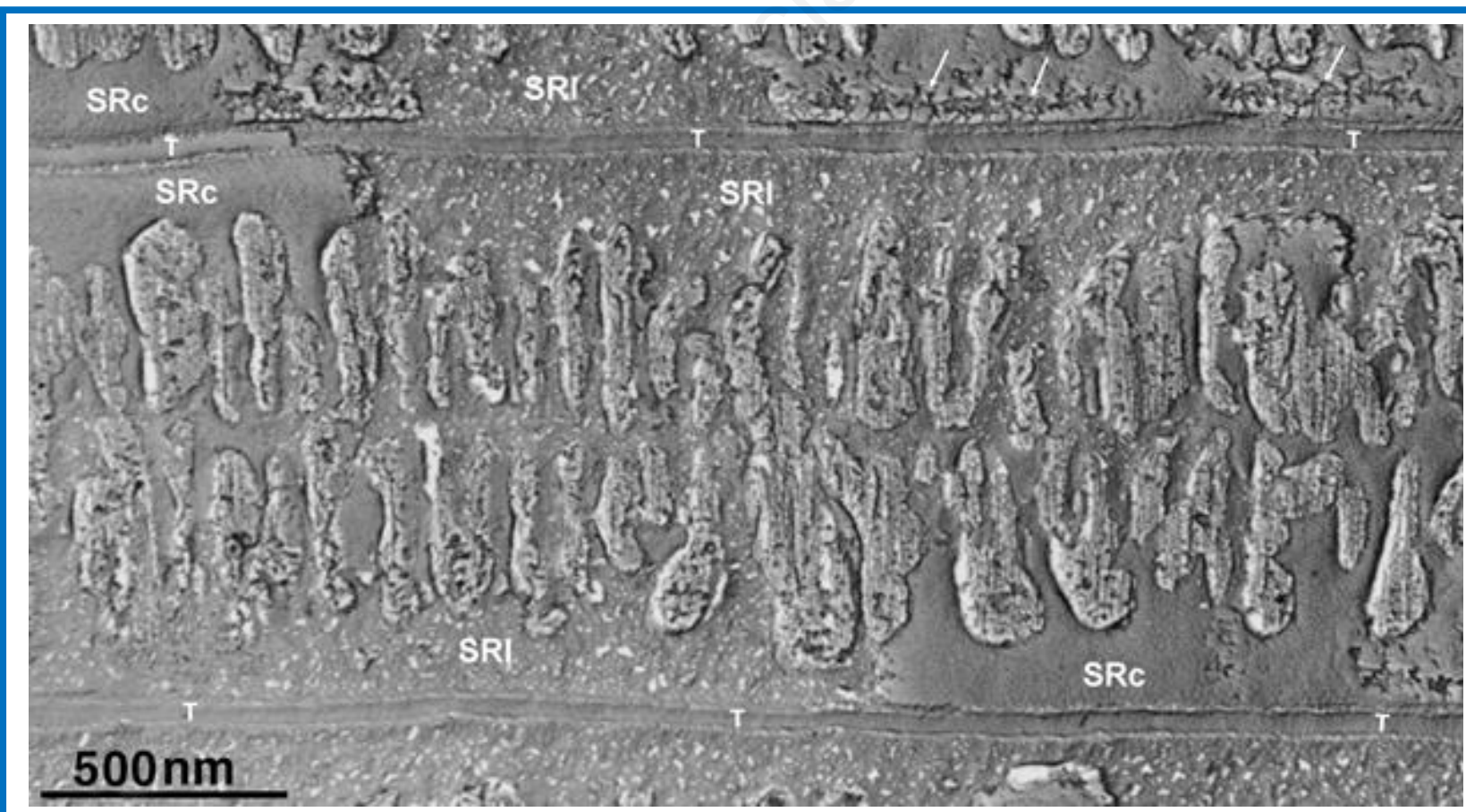

Fig 1. Freeze-fracture, deep-etch image from a body muscle of the glass fish. The SR and T tubules are at the upper surface and the underlying myofilaments are visible below. Two long uninterrupted T tubules (T) flanked by flat SR cisternae run transversely across the image. The cytoplasmic leaflet of the SR (SRc) shows bumps denoting location of the Ca-ATPase; the luminal leaflet (SRl) is broken up (an artefact of etching). Note continuity of the SR across the whole image: these are "continuous" triads. Calsquestrin (arrows) is visible within the exposed SR lumen. 


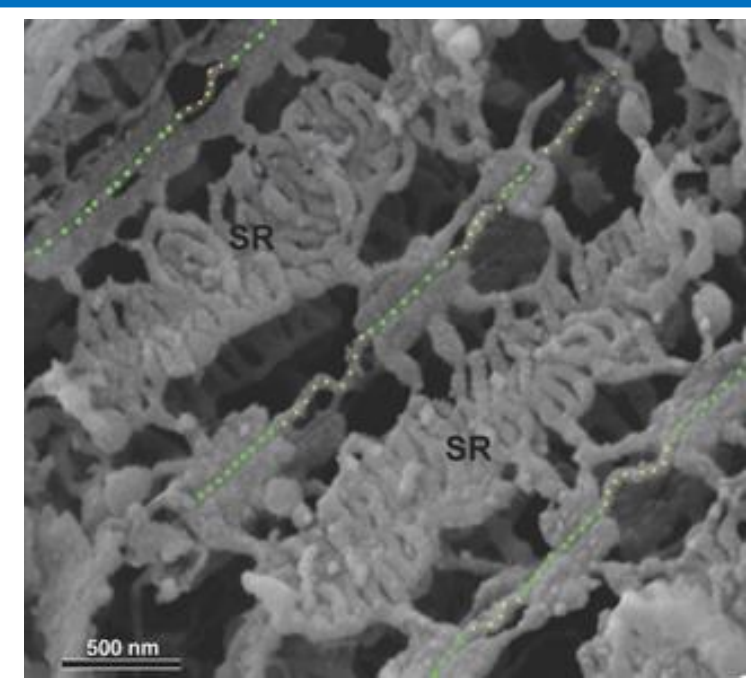

Fig 2. View of $S R$ and $T$ tubules in the lateral muscle of a small fresh water fish exposed after extraction of myofibrils by $\mathrm{OsO} 4$ maceration. The classical components of muscle membranes are easily recognized. The SR (SR) is arranged in extensive networks that are connected by longitudinal tubules to the flat junctional SR cisternae of discontinuous triads. The T tubules alternate between straight junctional segments (green dots) within triads and wavy free segments (yellow dots) between triads

were thus identified as derived from the red bundle. ${ }^{21}$ The clear structural dichotomy in content of mitochondria and structure of triads/T tubules between the central and peripheral fractures confirms that the identification was correct.

\section{Results}

We report that "white" fibers of small fish, constituting the bulk of the body muscles ${ }^{22}$ show an unusual triad organization in which the $\mathrm{T}$ tubule is constrained in its entirety between SR elements, so that no free $\mathrm{T}$ tubules are present, even though the muscles are not superfast. This is in contrast to the red fibers constituting the peripheral triangle close to the lateral line,${ }^{22}$ that show the more common pattern of free $\mathrm{T}$ tubule segments alternating with the junctional ones. Identification of the two regions was obtained not by pre-dissection of the muscles before fixing, but by clues in the samples prepared for microscopy (see supplemental information). Visualization of the SR/T relationships in a revealing manner was obtained by two technical approaches: freeze-fracture followed by deep etching and a relatively unknown maceration method that allows preservation of membrane systems after removal of all other cytoplasmic components. ${ }^{20,21}$

Figure 1 illustrates the extensive continuity of the SR coverage of $\mathrm{T}$ tubules in a freeze-fracture, deep-etch

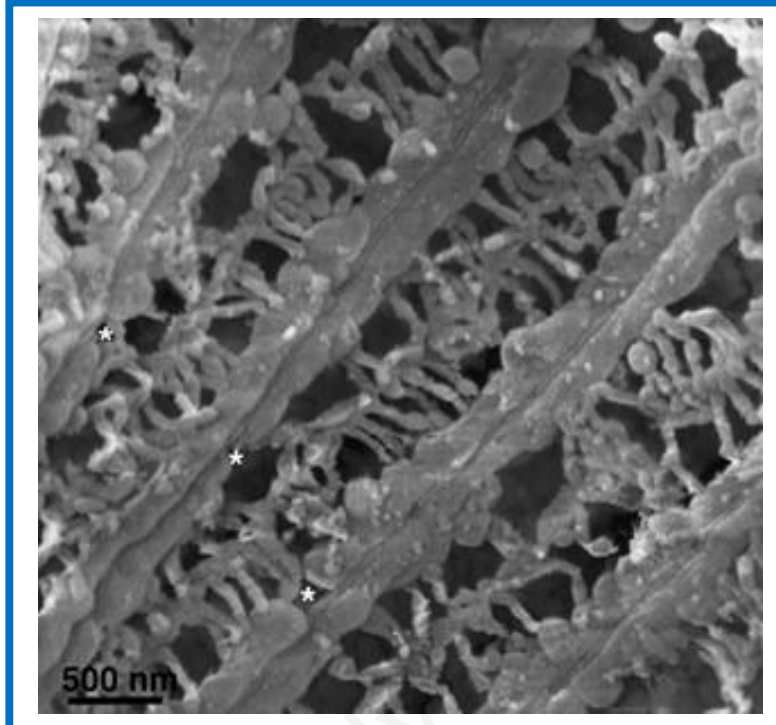

Fig 3. SR and T tubules in the main body muscle of the same fish as in Fig, 2. Several long triads (with a central T tubule) run obliquely across the image. The triad cisternae are mostly continuous, but occasional short gaps (asterisks) are present.

image from the white body muscles of a glass fish. $\mathrm{T}(\mathrm{T})$ tubules and triads from two $\mathrm{Z}$ line levels run transversely in the figure. Note continuity and straight course of the T tubules and the presence of continuous, uninterrupted SR (SR) profiles on either side of the $\mathrm{T}$ tubule, throughout the image and other areas of the same muscle (not shown). These are examples of "continuous triads" that are quite unusual.

Figure 2 shows a scanning electron micrograph of a red fiber at the periphery of a small fish, prepared by the maceration method. See supplemental information for identification. The extensive elongated membrane cisternae, mostly oriented longitudinally, belong to the sarcoplasmic reticulum. T tubules marked by colored dots cross the image diagonally. The empty spaces were occupied by myofibrils before the maceration process. Note two components of the T tubule. Some segments, marked by yellow dots, are free $\mathrm{T}$ tubules without direct connections to other membranes and with a wavy course. The other segments, marked by green dots, are junctional segments, with a straight path and flanked by two SR cisternae within triads. The alternation of discontinuous triads and free $\mathrm{T}$ tubules is the common disposition in the great majority of muscles.

Within the same fish (Figure 3) are areas in which long SR elements show only few discontinuities (labeled by asterisks). Due to the vagaries of the experimental approach, it was not possible to assign a specific position to this specific sample within the fish anatomy, but comparison with figure 4 suggests that it must belong to the main white body muscle. 


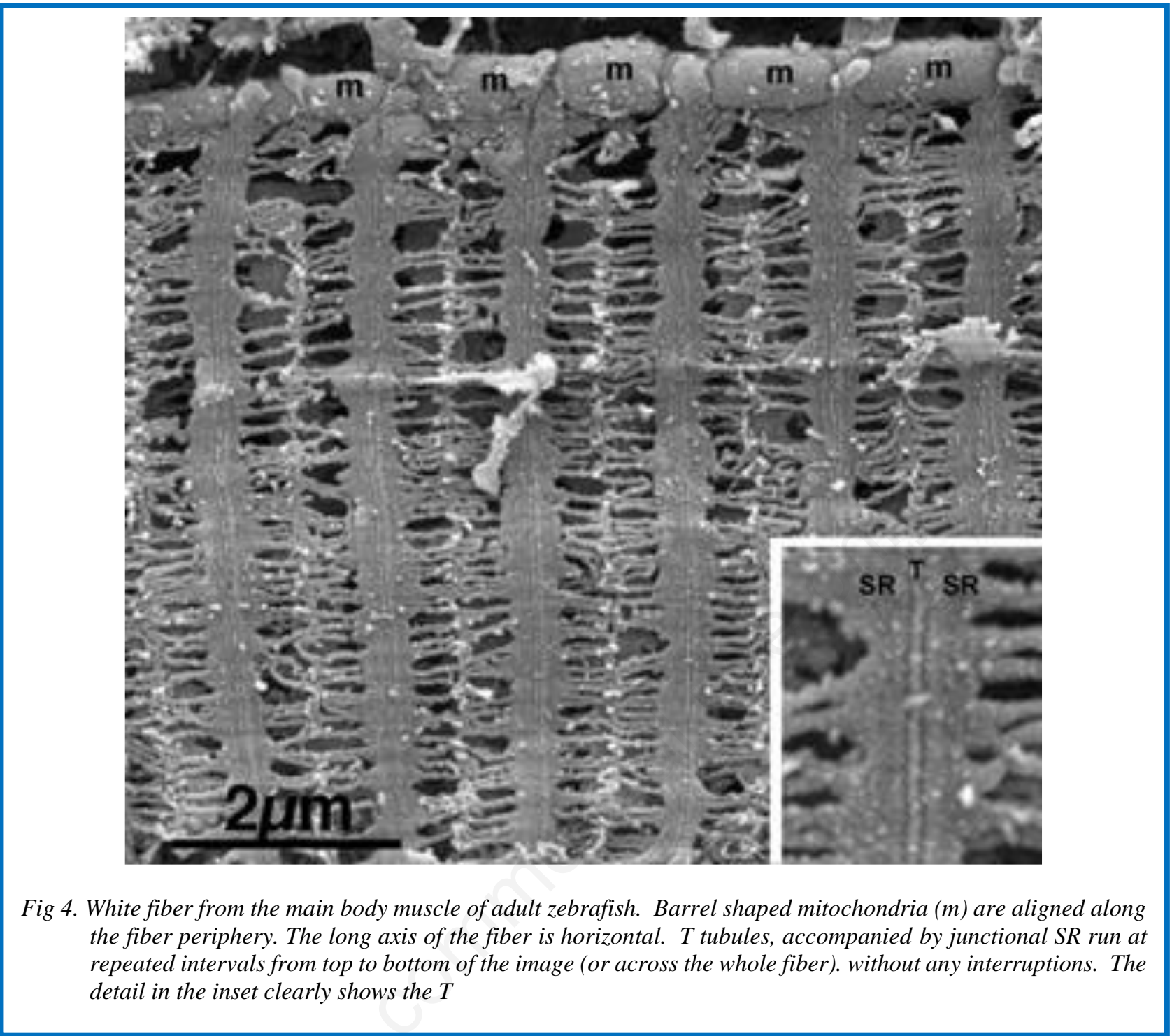

Figure 4 illustrates an SEM image from a fracture of a zebrafish. The fracture clearly involved the main white fiber region of the body (see supplemental information) and all samples show the same extensive flat regions of $\mathrm{SR}$ and $\mathrm{T}$ tubules that originally occupied the narrow spaces between the typical radially oriented myofibrils. The SR has the usual fence-like arrangement of longitudinal tubules alternated with the wider cisternae of the triads. Note that the triads can be traced without interruptions over the whole region and the inset clearly shows the narrow $\mathrm{T}$ tubule between the SR profiles. This is a disposition of continuous triads, comparable to that shown in figure 1 , that has been detected only in the main body muscle of fish.

\section{Discussion}

In the order to provide an interpretation of the continuous triad structure in the main body muscles of three small fish we propose a hypothesis based on the known fish physiology, combined with the fact that maintenance of constant fiber volume results in extension of the fiber cross sectional area when the fibers shorten, thereby leading to a stress of the triads. The hypothesis is simply that continuous triads can be maintained only in muscle fibers that are not normally subject to significant changes in sarcomere length. Muscle fiber behavior in the process of swimming, particularly as regards sarcomere length deviations, has been closely examined in a medium size fish. ${ }^{23}$ Considering the overall oblique disposition of muscle bundles in the main white fiber body and the bone curvature, the conclusion of that work is that white bundles of the main body musculature operate within a limited range of sarcomere lengths around maximum overlap even when recruited in an escape reaction. This being the case, the white fibers do not need a protection against stretching of the transversely oriented triad/T tubule network during activity. Hence a continuous triad is present. The red fibers of the lateral bundles, on the other hand, are subject to significant changes in sarcomere length and in cross sectional area during swimming. They depend on the presence of free, wavy 
T tubule segments in order to avoid damage to the triad structure. The hypothesis assigns to T tubules and to their interaction with the SR a function additional to the obvious e-c coupling role: the free tubules are given a role in protecting against damage to the membrane system in terms of a rupture of either triads or free $\mathrm{T}$ tubules that would be produced by stretching of the transverse network at very short sarcomere lengths. The result of such an interruption is expected to be a reduction of e-c coupling effectiveness, but not mechanical damage to the fiber. Mechanical damage to $\mathrm{T}$ tubule/triads has been proposed to explain the shallow ascending limb of the length tension curve that occurs at very short sarcomere lengths in vitro. Exploration of this possibility ${ }^{24}$ indicated inhomogeneous activation at very short sarcomere lengths. The concept is easily amenable to experimental confirmation based on further comparative observations. We therefore propose a challenge to the next serious electron microscopist: to establish whether in a given muscle fiber a relationship exists between extent of free $\mathrm{T}$ tubules and the extent of fiber shortening to be expected during normal activity. Any animal model may provide the answer, but muscles from an appropriately selected fish with demonstrated variations in extent of flexing during swimming would provide the best examples.

In conclusion, the observation that white body muscle fibers in three small fish show a previously unknown lack of free $\mathrm{T}$ tubule is consistent with the hypothesis that the function of free $\mathrm{T}$ tubules is to protect triads from stretch damage during shortening.

\section{List of acronyms}

e-c - excitation-contraction

fT - free tubule segments

jT - junctional tubule segments

SR - sarcoplasmic reticulum

$\mathrm{T}$ - transverse tubules

SJ - single-joint

\section{Authors contributions}

Manuela Lavorato performed experiments, Ramesh Iyer contributed to the manuscript, Clara Franzini-Armstrong contributed to and directed experiments, wrote the manuscript.

\section{Acknowledgments}

We thank Dr. Sally Page for insightful comments. This material was presented at the 2019 Spring Padua Muscle Days, University of Padova Botanical Garden, Aula Emiciclo March 30, 2019. ${ }^{25}$

\section{Funding}

Funded by Children's Hospital of Philadelphia Cardiac Center Grant to RI.

\section{Conflict of Interest}

The authors declare no conflicts of interest.

\section{Ethical Publication Statement}

We confirm that we have read the Journal's position on issues involved in ethical publication and affirm that this report is consistent with those guidelines.

\section{Corresponding Author}

Clara Franzini-Armstrong, Department of Cell and Developmental Biology, B42 Anatomy Chemistry Bldg. University of Pennsylvania Medical School, Philadelphia PA 19104, USA. Phone 5082747903

Email: armstroc@mail.med.upenn.edu

E-mails of co-authors

Manuela Lavorato: manuelalavorato@gmail.com Ramesh Iyer: iyerv1@email.chop.edu

\section{References}

1. Andersson-Cedergren E. Ultrastructure of motor end-plate and sarcoplasmic components of mouse skeletal muscle fiber. J Ultrastr Res 1959;suppl. 1:1-250.

2. Muscatello U, Andersson-Cedergren E, Azzone GF. The sarcotubular system of frog skeletal muscle. A morphological and biochemical study. J Biophys Biochem Cytol 1961;10(4 suppl):201-18.

3. Franzini-Armstrong C. Veratti and beyond: structural contributions to the study of muscle activation. Rend Fis Acc Lincei 2002;13:289-323.

4. Franzini-Armstrong C, Gilly WF, Aladjem E, Appelt D. Golgi stain identifies three types of fibers in fish muscle. J Muscle Res Cell Motil 1987;8:41827

5. Franzini-Armstrong C, Champ C, Ferguson DG. Discrimination between fast- and slow- twitch fibres of guinea pig skeletal muscle using the relative surface density of junctional transverse tubule membrane. J Muscle Res Cell Motil 1988;9:403-14.

6. Appelt, D, Shen V, Franzini-Armstrong C. Quantitation of Ca ATPase, feet and mitochondria in super fast muscle fibres from the toadfish, Opsanus tau. J Muscle Res Cell Motil 1991;12:54352.

7. Fawcett DW, Revel JP. The sarcoplasmic reticulum of a fast-acting fish muscle. J Biophys Biochem Cytol 1961;10 (4 suppl):89-109.

8. Nelson FE, Hollingworth S, Rome LC, Baylor SM. Intracellular calcium movements during relaxation and recovery of superfast muscle fibers of the toadfish swimbladder. J Gen Physiol 2014;143:60520.

9. Bass AH, Marchaterre MA. Sound-generating (sonic) motor system in a teleost fish (Porichthys notatus): Sexual polymorphism in the ultrastructure of myofibrils. J Comp Neurol 1989;286:141-53.

10. Lewis MK, Nahirney PC, Chen V, et al. Concentric intermediate filament lattice links to specialized Zband junctional complexes in sonic muscle fibers of 


\section{Role of non-junctional transverse tubules}

Eur J Transl Myol 29 (2): 86-91, 2019

the type I male midshipman. J Struct Biol 2003;143:56-71.

11. Rome LC. Design and function of superfast muscles: New insights into the physiology of skeletal muscle. Annu Rev Physiol 2006;68:193221.

12. Rome LC, Syme DA, Hollingworth $\mathrm{S}$ et al. The whistle and the rattle: the design of sound producing muscles. Proc Nat Acad Sci 1996;93:8095-100.

13. Schaeffer PJ, Conley KE, Lintstedt SL. Structural correlates of speed and endurance in skeletal muscle: the rattlesnake tailshaker muscle. J Exp Biol 1996:199:351-58.

14. Revel JP. The sarcoplasmic reticulum of the bat cricothyroid muscle. J Cell Biol 1962;12:571-88.

15. Franzini-Armstrong C. Electron Microscopy: From 2D to 3D Images with Special Reference to Muscle. Eur J Transl Myol 2015;25(1):4836. doi: 10.4081/ejtm.2015.4836. eCollection 2015 Jan 7.

16. Samsó M. 3D Structure of the Dihydropyridine Receptor of Skeletal Muscle. Eur J Transl Myol 2015;25:4840. doi: 10.4081/ejtm.2015.4840. eCollection 2015 Jan 7.

17. Wagenknecht T, Hsieh C, Marko M. Skeletal Muscle Triad Junction Ultrastructure by FocusedIon-Beam Milling of Muscle and Cryo-Electron Tomography. Eur J Transl Myol 2015;25:4823. doi: 10.4081/ejtm.2015.4823. eCollection 2015 Jan 7.

18. Baker MR, Fan G, Serysheva II. Single-Particle Cryo-EM of the Ryanodine Receptor Channel in an Aqueous Environment. Eur J Transl Myol 2015;25:4803. doi: 10.4081/ejtm.2015. 4803. eCollection 2015 Jan 7.

19. Jayasinghe ID, Clowsley AH, Munro M, et al. Revealing T-Tubules in Striated Muscle with New
Optical Super-Resolution Microscopy Techniques. Eur J Transl Myol 2015;25:4747. doi: 10.4081/ ejtm.2015. 4747. eCollection 2015 Jan 7.

20. Tanaka K, Mitsushima A. A preparation method for observing intracellular structures by scanning electron microscopy. J Microsc (London) 1984;133:213-22.

21. Ogata T, Yamasaki Y. High-resolution scanning electron microscopic studies on the threedimensional structure of the transverse-axial tubular system, sarcoplasmic reticulum and intercalated disc of the rat myocardium. Anat Rec 1990;228:277-87.

22. Franzini-Armstrong C, Porter KR. Sarcolemmal invaginations constituting the $\mathrm{T}$ system in fish muscle fibers. J Cell Biol 1964;22:675-96.

23. Rome LC, Sosnicki AA. Myofilament overlap in swimming carp. II. Sarcomere length changes during swimming. Am J Physiol 1991;260:C28996.

24. Julian FJ, Morgan DL. Tension, stiffness, unloaded shortening speed and potentiation of frog muscle fibres at sarcomere lengths below optimum. J Physiol 1981;319:205-17.

25. Carraro U. Collection of the Abstracts of the 2019Sp PMD: Translational Myology and Mobility Medicine. Eur J Transl Myol 2019;29(1):8155. doi: 10.4081/ejtm.2019.8155. eCollection 2019 Jan 11.

Submissions: May 03, 2019

Revision received: May 13, 2019

Acceptance: May 13, 2019 